\title{
Advances in Radioembolization - Embolics and Isotopes
}

\author{
Joshua Burrill', Urs Hafeli² and David M Liü4,4*
}

${ }^{1}$ Department of Radiology, St Pauls Hospital, Vancouver, British Columbia, Canada

${ }^{2}$ Faculty of Pharmaceutical Sciences, University of British Columbia, Vancouver, British Columbia, Canada

${ }^{3}$ Department of Radiology, Vancouver General Hospital|University of British Columbia, Vancouver, BC, Canada

${ }^{4}$ Angio/interventional Section, UCLA Department of Radiological Sciences, David Geffen School of Medicine, University of California Los Angeles, Los Angeles, CA

\begin{abstract}
Selective internal radiation therapy (SIRT), otherwise known as radio embolization is now becoming a common procedure performed for those patients with primary hepatic neoplasia [such as hepatocellular carcinoma], and liver dominant metastatic disease [such as in near endocrine disease, and colorectal carcinoma]. The current technology platforms incorporate the use of yttrium 90, a pure beta emitter loaded on either a resin microsphere, or ceramic microsphere.
\end{abstract}

Although clinical outcomes have been encouraging with both technology platforms, second-generation radioembolic devices [utilizing either new processes of microsphere synthesis, or different radioactive isotopes] are currently under development, or clinical study. The purpose of this manuscript is to provide the reader with some perspectives regarding the next generation of radioembolic devices, and discussing the advantages and disadvantages of both current, and future platforms.

\section{Introduction}

It is estimated that more than 1 million people are diagnosed with primary or secondary liver malignancy each year [1]. Secondary hepatic metastases (including those from the gastrointestinal tract, breast, and neuroendocrine tumours) are common; with $60 \%$ of patients with colorectal carcinoma develop liver metastases [2]. Primary liver cancer including hepatocellular carcinoma (HCC) and cholangiocarcinoma, is the sixth most common cancer worldwide with an abysmal five year survival of $3-5 \%$ [1].

Radiation therapy using direct external beam irradiation has been used to treat both HCC and liver metastases, with limited results. Partial response with symptomatic improvement in the treatment of HCC was demonstrated back in the 1970s [3], limited by the inability to provide whole liver irradiation in an effective manner, with documented radiation induced liver disease (presenting in a fashion similar to venooclusive disease), so termed RILD developing at exposure levels as low as $30-35$ Gy (RILD) [4]. This dose, resulting in a 5\% incidence of RILD, is well short of the exposure required to ellicit tumorocidal effect.

Intra-arterial radiotherapy of liver cancer is not a new concept having been first attempted in the 1960s with yttrium-90 microspheres with encouraging response within the neuroendocrine population [5]. Selective internal radiation therapy (SIRT), taking advantage of the preferential hepatic arterial supply of liver neoplasms experienced technical limitiations, predominantly due to challenges of dosimetry, non-selective injection of microspheres (injected at the level of the celiac artery), and leaching, that has not been described in reference to current commercialized products [2].

The evolution of more advanced dosimetric techniques, supraselective hepatic arterial catheterization, awareness of the importance of hepatic-gastric and pancreatic anastamoses, measurement of hepatopulmonary shunting, and stable embolic platforms with minimal leaching have acted to improve the ratio of tumour to liver/ rest of the body dose.

\section{Radioisotopes}

Radioembolization uses an active radioisotope combined with an embolic delivery platform. Various radioisotopes have been used including yttrium-90 (Y-90), iodine-131 (I-131), rhenium-188 (Re188), and holmium-166 (Ho-166). These are all $\beta$ emitters, with $\gamma$ emission from Re-188, Ho-166, and I-131.

The tissue penetration of $\beta$ particles (electrons) is from a few millimetres up to $1 \mathrm{~cm}$ which reduces the dose to the normal liver when combined with the appropriate embolic. As current methods in yttrium 90 microparticle manufacture require access to facilities they can perform neutron bombardment, the logistics involved in not only the manufacturing, but also the rapid transportation due to its relatively short half-life] provide significant challenges in both the manufacture, and transportation of the radial embolic material. As a result of the geographic distribution of manufacturing facilities, and clinical sites, high variation in the specific activity per particle may occur as a result of decay kinetics at the time of transportation, or variations in the dose calibration techniques.

Other radioisotopes, including phosphorus-32, copper-64, zirconium-89, fluorine-18, and yttrium-86, have all been investigated as possible sources for either SIRT or dosimetry, instead of technetium$99 \mathrm{~m}$ however have encountered challenges. For example decayed SIRSpheres have be loaded with F-18 produce in a cyclotron. Problems occurred due to substantial in-vivo leaching in a rat model [7].

\section{Ideal Radioisotope}

\section{Half-life of hours}

Easily synthesized; can be loaded with radioactivity close to the

*Corresponding author: David Liu, Department of Radiology, Vancouver Genera Hospital|University of British, 855 W 12th Ave, JP Pavilion G873, Vancouver, British Columbia, Canada, V5Z 1M9, E-mail:dave.liu@vch.ca

Received April 26, 2011; Accepted May 26, 2011; Published June 15, 2011

Citation: Burrill J, Hafeli U, Liu DM (2011) Advances in Radioembolization - Embolics and Isotopes. J Nucl Med Radiat Ther 2:107. doi:10.4172/2155-9619.1000107

Copyright: ( 2011 Burrill J, et al. This is an open-access article distributed under the terms of the Creative Commons Attribution License, which permits unrestricted use, distribution, and reproduction in any medium, provided the original author and source are credited. 
facility in which implantation is to occur

High energy particle with low mean free path e.g. $\beta$

Detectable percentage of $\gamma$, or positron emission for imaging

Safe decay product: Biocompatible and bioabsorbable

Cheap local manufacture

No leaching, and carrier free suspension, minimizing hematogenous and systemic exposure

\section{Embolics}

It has been well established that tumor vascularity within the hepatic circulation is a complex anatomical structure, consisting of the vascular plexus of abnormal blood vessels ranging in size from 25 to $75 \mu \mathrm{m}$. These abnormal blood vessels have been targeted through various embolic methods, including starch particles, albumin, polyvinyl alcohol, gelatin, ethiodol, glass and resin. Recent literature utilizing super paramagnetic iron oxide (SPIO) loaded particles demonstrate improved penetration into tumor vascular plexus with smaller size particles (as small as $100 \mu \mathrm{m}$ ) [8], however particles smaller than $40 \mu \mathrm{m}$ had demonstrated significant pulmonary shunt tumor perfusion [9]. This is abnormal bypassing of the liver and tumor, with non-targeted embolization of the lungs. Commercial radioembolic products currently are produced in the 30 to $70 \mu \mathrm{m}$ range, with a variance in the particles size to allow for deposition and distribution into various tumor vessel sizes, permitting a more even distribution.

\section{Ideal embolic platform}

Isodense with blood

Stable embolic/radioisotope ligand with no leaching

Ease of production

\section{Consistent size}

Can be utilized with a pure $\gamma$ emitter for dosimetry and estimation of hepatopulmonary shunt fraction

Particle or material utilized for mesenteric angiography, and pollination perfusion determination should behave in a similar fashion to the therapeutic radiomicrosphere

Bioabsorbable polymer or substrate with a half life at least $7 \mathrm{x}$ longer that the radioisotope

\section{Radioembolic Platforms}

\section{Yttrium-90 (commercially available)}

$\beta$ emitter: average $933.7 \mathrm{KeV}, \max 2.28 \mathrm{MeV}$

\section{Half life: 64.2 hours}

Average tissue penetration: $2.5 \mathrm{~mm}$

X90* : $5.2 \mathrm{~mm}$ (radius of the sphere in which $90 \%$ of the energy emitted from a point source is absorbed.) Method of manufacture: Neutron activation of stable Y-89 in a nuclear reactor.

$\begin{array}{cll} & \text { SIR-Spheres } & \text { Theraspheres } \\ \text { Type of microsphere } & \text { Resin } & \text { Glass } \\ \text { Diameter }(\mu \mathrm{m}) & 32 \pm 10 & 25 \pm 10 \\ \text { Specific gravity }+ & 1.6 & 3.7 \\ \text { Specific activity }(\mathrm{Bq} / \mathrm{MS}) & 40 & 2467 \wedge\end{array}$

$\begin{array}{lll}\text { Mean no spheres / dose } & 20 \times 10^{6} & 4 \times 10^{6} \\ \text { Patient dose (GBq) } & 0.5-3 & 3-20\end{array}$

$\wedge$ at time of manufacture

+ Specific gravity of Blood: 1.05 [10]

\section{SIR-SPHERES (Sirtex Medical Inc, Sydney, Australia)}

In a cohort study looking at 110 patients with liver metastases from various primaries treated with SIR-Spheres SIRT the average survival was 323 days (10.6 months). 350 day survival was $55 \%$ for colorectal metastases and 39\% for breast carcinoma metastases [11]. Complications included three cases of cholecystitis, six of gastritis and one hepatic failure. Post-embolization syndrome occurred in approximately two-thirds of patients. A phase III randomized trial comparing fluorouracil chemotherapy with and without SIRT in 44 patients with colorectal metastases showed a significantly better time to tumor progression (TTP) with SIRT. Survival was also better but not significantly, however there was substantial crossover with 10 of 23 fluorouracil patients receiving SIRT [12]. Similar findings have been seen in other studies $[13,14]$.

SIR-Spheres have been used to treat 71 patients with unresectable HCC [15]. Median survival was 9.4 months, and in two cases there was complete histological response suggesting this can be curative. $16 \%$ had post-embolization syndrome, but no cases of radiation pneumonitis or hepatitis were recorded. A similar survival has been seen elsewhere [16]. SIR-Spheres have been used to treat HCC with portal vein thrombosis, with no significant liver toxicity and a median survival of 10.1 months [17]. Data collected on a cohort of 515 patients treated with SIR-Spheres for unresectable liver tumors showed that 5\% (28 patients) died from RILD. Out of the 680 treatments, 79 were for HCC and thee HCC patients died of RILD [18].

\section{Advantages}

- Lower specific activity allows for more uniform distribution of radioactivity within tumor.

- Dose arrives as a parent dose, allowing for multiple fractionated doses to be drawn per patient.

- Delivery device allows for intermittent administration of contrast to assess blood flow.

- Lower specific gravity may allow for more uniform, flow directed deposition of microspheres.

- Pure Y-90 radioactive species without mutant radioactive species.

- 'carrier free' suspension, resulting in minimal systemic exposure.

- extensively published clinical outcome literature in the context of metastatic colorectal carcinoma, neuroendocrine disease, hepatocellular carcinoma.

\section{Disadvantages}

- standardized body surface area [BSA] dose activity model may result in under demonstration of targeted dose in situations of large bulky tumors

- Dose administration Kit is designed primarily for safety, however can be somewhat cumbersome during administration

- lower specific activity of particles may result in stasis, or sluggish antegrade flow prior to full dose administration

\section{THERASPHERE (MDS Nordion, Ottawa, Ontario, Canada)}

Theraspheres have been used in a number of studies looking at the treatment of HCC with and without portal vein thrombosis (PVT). One study of 118 patients showed a median survival of 15.3 months 
for patients without PVT and 4.4 months with main PVT [19], which is better than seen with I-131-Lipiodol [20]. There were few complications with no cases of radiation pneumonitis or gastrointestinal ulceration. In a retrospective review of patients with HCC treated with Therasphere SIRT or mitomycin-cisplatin-adriamycin-lipiodol chemoembolization there was a longer TTP and less toxicity with SIRT [21]. A survival benefit was not demonstrated. Similar toxicity and TTP findings were seen with another cohort study from Germany looking at 159 cases [22]. This study suggested that the median survival after SIRT of 16.4 months was better than in the SHARP trial for sorafenib (10.7 months). Theraspheres have also been used to downstage HCC prior to transplantation or resection, with a significant improvement in the percentage downstaged from $\mathrm{T} 3$ to $\mathrm{T} 2$ when compared to chemoembolization (58\% versus $31 \%$ ). A study has looked at using extended-shelf-life Theraspheres to increase the number of particles used and therefore increase the distribution and reduce the risk of a severe response from non targeted embolization [23].

\section{Advantages}

- High specific activity allows for complete administration of partition modeled dose activity.

- Lower risk of non-targeted embolization due to high specific activity and high specific gravity per microsphere.

- Pre administration and calibration of dose activity prior to administration is a single step process.

- Extensive published literature demonstrating positive outcome for use in hepatocellular carcinoma.

- Current dose administration kit intuitive, and easy to use.

\section{Disadvantages}

- Dose must be delivered at a specific day and time.

- Precalibrated vials contain radioactivity that cannot be divided or fractionated.

- May result in under distribution of microspheres in larger tumors resulting in 'swiss cheese' response, and non-uniform microdosimetry.

- High specific gravity may cause settling or migration of microspheres.

- Due to higher specific activity, non-targeted embolization may result in more severe response (e.g. radiation cholecystitis )

- Unable to check for reflux or stasis during administration [this is especially relevant in extended decay strategies such as Therasphere EX)

- Inherent non-intended radioactive species present in matrix ( e.g. Y-88 half-life 107 days, Europium-154 half-life 8.8 y)

\section{Iodine-131}

$\beta$ emitter: mean $192 \mathrm{KeV}, \max 610 \mathrm{KeV}$

$\gamma$ emitter: $364 \mathrm{KeV}$

Half life: 8.04 days

Average tissue penetration: $0.4 \mathrm{~mm}$

X90: $0.7 \mathrm{~mm}$ (radius of the sphere in which $90 \%$ of the energy emitted from a point source is absorbed.) Method of manufacture: Isotope exchange after formation in a nuclear reactor.

\section{Patient dose: 0.9 - 2.4 GBq}

Iodine-131 had been used to label lipiodol, a mixture of iodized esters of poppyseed oil fatty acids. It forms an emulsion of fat droplets with a diameter of $20-200 \mu \mathrm{m}$. I-131-lipiodol has been used for the treatment of HCC in cases with and without portal vein thrombosis, and for the treatment of liver metastases. The procedure involves a selective hepatic artery injection of 2 to $3 \mathrm{ml}$ of I-131-lipiodol with an activity of 0.9 to $2.4 \mathrm{GBq}$ [24,25]. Biodistribution studies using low dose I-131-lipiodol showed that it is almost exclusively retained by the liver and the lungs with a greater liver to liver+lung ratio for HCC (mean $76 \%$ ) than for liver metastases (mean $86.2 \%$ ). However the tumor to non-tumor ratio was higher in the HCC group $(4.3 \pm 2.6)$ than for the liver metastases group $(2.4 \pm 0.7)$ [26]. This high lung uptake may explain lung fibrosis which occurs in around $2 \%$ of patients and is fatal in about half of those. It is the most serious complication of treatment with I-131-lipiodol [20].

In a randomized study looking at the treatment of HCC and portal vein thrombosis with I-131-lipiodol versus best supportive care there was a $71 \%$ versus $10 \% 3$ month survival, despite a uniformly poor long term survival of $7 \%$ and $0 \%$ at 9 months respectively [27]. Another randomized study compared HCC chemoembolization and SIRT with I-131-lipiodol. There was a similar overall survival at 1 and 2 years (38.5\% and $22 \%$ for chemoembolization, and $42 \%$ and $22 \%$ for I- 131 , respectively), but with significantly less side effects in the SIRT group [28]. As a post-surgical adjuvant after potential curative resection for HCC, I-131-lipiodol has been shown to significantly improve overall and disease-free survival for more than 5 years after surgery (overall survival at 5 years $66.7 \%$ versus 36.4\%) [29]. I-131-lipiodol has been used to treat colorectal carcinoma metastases combined with chemotherapy resulting in an objective response in two out of three of the patients [1]. No significant reduction in the size of liver metastases was seen in one small study although there was a clear reduction in abdominal pain which was thought to be tumour-related [25].

\section{Advantages}

-Easy administration.

- Gamma emission enables post-procedural imaging.

- High tumor to non-tumor uptake by HCC.

- Can be used to treat patient with portal vein thrombosis due to low embolic load.

- No collateral arterial embolization needed prior to radioembolization.

- Product can be created from readily available supplies within a hospital, resulting in lower cost per session.

\section{Disadvantages}

- Long half life means that patient requires hospitalization for a week for radioprotection.

- High $\gamma$ energy.

- Short $\beta$ range.

- Lung fibrosis resulting in death in approximately $1 \%$ of cases.

- No significant tumor reduction seen in the treatment of liver metastases.

- May require multiple sessions to achieve maximum response.

\section{Rhenium-188}

$\beta$ emitter: mean $764 \mathrm{KeV}, \max 2.1 \mathrm{MeV}$

$\gamma$ emitter: $155 \mathrm{KeV}$

Half life: 16.9 hours

Average tissue penetration: $3.8 \mathrm{~mm}$

X90: $1.9 \mathrm{~mm}$ (radius of the sphere in which $90 \%$ of the energy emitted from a point source is absorbed.) Method of manufacture: W-188 / Re-188 generator possible on site.

$\begin{array}{lll}\text { Diameter }(\mu \mathrm{m}) & \text { Re-188-HSAM } & \text { Re-188-PLA } \\ 25(14-40) & 30 \pm 1\end{array}$


Specific gravity

Specific activity (Bq / MS)-

Mean no spheres / dose $2-3 \times 10^{5}$

Patient dose (GBq)
1.28

up to 20000

$8 \times 10^{6}$

$3-20$

Rhenium-188 has been used with glass microspheres, human serum albumin microspheres (HSAM), poly (L-lactide) (PLA) microspheres, and lipiodol as embolic platforms. A four to five fold higher Re-188 activity is required to obtain an equivalent absorbed dose as Y-90 [2]. Some initial studies of Re-186/188 glass microspheres were not taken further due to the disadvantages of requiring neutron activation in a nuclear reactor, dual activity and high density [30].

\section{Re-188-Lipiodol}

Re-188 has been conjugated with lipiodol using 4-hexadecyl-1,2,9,9tetramethyl-4,7-diaza-1,10-decanethiol (HDD) and used to treat HCC. Re-188 has obvious advantages over I-131 due to less $\gamma$ emission, greater $\beta$ penetration, and reduced cost. One and two year survival is similar to I-131-Lipiodol, $46 \%$ and $23 \%$ respectively [31]. Disadvantages of Re-188-lipiodol include urinary excretion of metabolites resulting in a loss of $44.1 \%$ (mean) of the administered activity, a low radiochemical yield, and lung fibrosis, also seen with I-131-lipiodol [32].

\section{Re-188-HSAM}

Re-188 has been added to HSAM using a W-188/Re-188 generator with $10 \%$ leaching after 30 hours incubation [33]. Re-188-HSAM has been used to treat HCC and colorectal liver metastases in 10 patients [34]. Tc-99m-HSAM was used to determine the treated volume of the liver and work out applied activity. In this limited study it was only possible to treat the entire tumor mass in two of the 10 patients as selective angiographic administration was used. Despite this, 1 year survival was $40 \%$ and either partial remission or stable disease was seen in $70 \%$. A mean urinary excretion rate of $8.9 \%$ of the injected activity was measured within 72 hours. There was no RILD-related fatality despite a single patient with grade three liver toxicity. A further unpublished phase 2 clinical trial had 22 patients. At 3 months either partial response or stable disease was seen in $89 \%$ by RECIST criteria, and $78 \%$ clinically [35].

\section{Re-188-PLA}

Tc-99m labeled PLA microspheres have been manufactured and used to perform lung perfusion imaging as a proof of concept study prior to labeling with Re-188 [36]. These microspheres are manufactured with a high degree of accuracy allowing the diameter to be sized within less than $+/-5 \%$. Re-188 and Tc-99m have a similar chemistry and studies are ongoing looking at the in-vivo performance of Re-188-PLA. Currently under active investigation, however this technology platform has not been attempted in humans.

\section{Advantages}

- $\beta$ and $\gamma$ emission allow post-procedural dosimetry.

- Produced on site using a low-cost Tungsten-188 / Re-188 generator.

\section{Disadvantages}

- Greater patient dose required due to its shorter half-life.

\section{Holmium-166}

$\beta$ emitter: mean $670 \mathrm{KeV}$, max $1.85 \mathrm{MeV}$

$\gamma$ emitter: $80.6 \mathrm{KeV}$

Half life: 26.8 hours

Average tissue penetration: $2.2 \mathrm{~mm}$

X90: $2.1 \mathrm{~mm}$ (radius of the sphere in which $90 \%$ of the energy emitted from a point source is absorbed.) Method of manufacture: Neutron activation in a nuclear reactor.

$\begin{array}{ll} & \text { Ho-166-PLA } \\ \text { Diameter }(\mu \mathrm{m}) & 30 \pm 5 \\ \text { Specific gravity } & 1.4 \\ \text { Specific activity (Bq / MS) } & \text { up to } 450 \\ \text { Mean no spheres / dose } & 50 \times 10^{6}\end{array}$

Holmium-166 has been combined with a PLA embolic platform and chitosan embolic platform. It has a lower energy and shorter half life than Y-90 and therefore a lower absorbed dose requiring three times more radioactivity than Y-90 [2].

\section{Ho-166-PLA}

No human studies have been performed using Ho-166-PLA, however animal studies have been performed to assess biodistribution of the microspheres using pigs with no postembolization syndrome [37]. A study with VX2 carcinoma implanted rabbits with Ho-166-PLA injected into the hepatic artery showed an arrest in tumour growth [2]. Low leaching occurs with a cumulative release in vitro $0.7 \%$ in a phosphate buffer after 52 weeks. The HEPAR phase 1 clinical trial is ongoing [38].

\section{Ho-166-Chitosan}

Ho-166-Chitosan dissolves in water under acidic conditions but forms a solid under neutral or basic conditions. A study has looked at treating single HCC in 54 patients [39]. Serum alkalinization was necessary to reduce the amount of leaching into the systemic circulation. Partial or complete response occurred in $78 \%$ of patients, with a median progression free survival of 34 months and a one year overall survival of approximately $88 \%$. There was significant toxicity with transient RILD in $26 \%$ of patients and two fatalities due to infection and hepatoma rupture. Transient hematological abnormalities occurred in up to $28 \%$ of patients. It is important to note that this study was performed on patients who were suitable for resection unlike most other Y-90 studies.

\section{Advantages}

- High x-ray attenuation and well imaged with fluoroscopy.

- Paramagnetic and therefore visualized by MRI.

- Low leaching as PLA microspheres.

- Biodegradability of both PLA and chitosan.

\section{Disadvantages (Ho-166-Chitosan)}

- Supraselective catheterization required.

- Serum alkalinization needed to avoid leaching.

- Difficult determination of microdosimetry.

- Low therapeutic index.

\section{Radioembolization Protocol}

Standard SIRT is a two stage process. The first stage involves an angiogram to map out and embolize the branches of the hepatic artery supplying non-hepatic tissue. An injection of, usually, technetium-99 microaggregated albumin (Tc-99m-MAA) is used to calculate the proportion of hepatopulmonary shunting and to optimize and exclude significant gastrointestinal uptake. The target dose can be calculated a number of ways but depends on the degree of shunting, which can also be a contraindication to the procedure. The aim is to keep the dose to the lungs below 30 Gy while delivering a dose of $120 \pm 20$ Gy to the tumour if utilizing a partition model, or alternatively body surface area [BSA formulation] [20]. 


\section{Conclusions}

Over 20,000 radioembolization therapies have been performed to date, utilizing the above technology platforms. In general objective imaging-based response has been excellent, with lower side effect profiles, when administered correctly, as compared to bland embolization and chemoembolization. Each of the discussed platforms possesses unique benefits and limitations. Despite these challenges, several phase III randomized control trials, predominantly with resin Y-90 microspheres, have been established, e.g., SIRFLOX, FOXFIRE, SORAMIC. These have been specifically powered to determine if incorporation of radioembolization offers overall survival benefit and/or progression free survival benefit in the HCC and metastatic colorectal carcinoma populations. Preliminary results are expected within the next 2-3 years.

Despite the unequivocal success of the therapy, many aspects of radioembolization remain challenging. These include determination of embolic distribution, microdosimetry, optimization of specific activity, active loading of specific activity per sphere and post implantation dosimetry. All aspects of current clinical and pipeline therapeutics serve to address some if not all of these challenges. This paves the way for second generation technologies, allowing for a more predictable administration and reliable response.

\section{Acknowledgement}

J. Cwikla, Department of Radiology, Hospital Ministry of Internal Affairs \& Administration, Warsaw. For use of their unpublished data

\section{References}

1. Bult W, Vente MA, Zonnenberg BA, Van Het Schip AD, Nijsen JF (2009) Microsphere radioembolization of liver malignancies: current developments. $Q$ J Nucl Med Mol Imaging 53: 325-335.

2. Chan R, Kerr D (2004) Hepatic arterial chemotherapy for colorectal cancer liver metastases: a review of advances in 2003 Curr Opin Oncol16: 378-384

3. Di Bisceglie AM, Rustgi VK, Hoofnagle JH, Dusheiko GM, Lotze MT (1988) Hepatocellular carcinoma. Ann Intern Med108: 390-401.

4. Lawrence TS, Robertson JM, Anscher MS, Jirtle RL, Ensminger WD, (1995) Hepatic toxicity resulting from cancer treatment. Int $\mathrm{J}$ Radiat Oncol Biol Phys 31: $1237-1248$

5. Ariel IM (1965) Treatment of inoperable primary pancreatic and liver cancer by the intra-arterial administration of radioactive isotopes. Ann Surg 162: 267-278.

6. Lau WY, Leung WT, Ho S, Leung NW, Chan M, et al. (1994) Treatment of inoperable hepatoceliular carcinoma with intrahepatic arterial yttrium-90 microspheres: a phase I and II study. Br J Cancer 70: 994-999.

7. Selwyn RG, Avila-Rodriguez MA, Converse AK, Hampel JA, Jaskowiak CJ et al. (2007) 18F-labeled resin microspheres as surrogates for 90Y resin microspheres used in the treatment of hepatic tumors: a radiolabeling and PET validation study. Phys Med Biol 52: 7397-7408.

8. Namur J, Chapot R, Pelage JP, Wassef M, Langevin F, et al. (2007) MR imaging detection of superparamagnetic iron oxide loaded tris-acryl embolization microspheres. J Vasc Interv Radiol18: 1287-1295.

9. Bastian P, Bartkowski R, Köhler H, Kissel T (1998) Chemo-embolization of experimental liver metastases. Part I: distribution of biodegradable microspheres of different sizes in an animal model for the locoregional therapy. Eur J Pharm Biopharm 46: 243-254

10. Trudnowski RJ, Rico RC (1974) Specific gravity of blood and plasma at 4 and 37 degrees C. Clin Chem 20: 615-616.

11. Cianni R, Urigo C, Notarianni E, Saltarelli A, D’Agostini A, (2010) Radioembolisation using yttrium 90 (Y-90) in patients affected by unresectable hepatic metastases. Radiol Med 90: 619-633.

12. Hendlisz A, Van den Eynde M, Peeters M, Maleux G, Lambert B, et al. (2010) Phase III trial comparing protracted intravenous fluorouracil infusion alone of with yttrium-90 resin microspheres radioembolization for liver-limited metastatic colorectal cancer refractory to standard chemotherapy. J clin oncol 28: 3687 3694.

13. Van Hazel G, Blackwell A, Anderson J, Price D, Moroz P, et al. (2004) Randomised phase 2 trial of SIR-Spheres plus fluorouracil/leucovorin chemotherapy versus fluorouracil/leucovorin chemotherapy alone in advanced colorectal cancer. J Surg Oncol 88: 78-85

14. Gray B, Van Hazel G, Hope M, Burton M, Moroz P, (2001) Randomised trial of SIR-Spheres ${ }^{\circledR}$ plus chemotherapy vs. chemotherapy alone for treating patients with liver metastases from primary large bowel cancer. Ann Oncol 12: 1711 1720.

15. Lau WY, Ho S, Leung TW, Chan M, Ho R, et al. (1998) Selective interna radiation therapy for nonresectable hepatocellular carcinoma with intraarteria infusion of 90Yttrium microspheres. Int J Radiat Oncol Biol Phys 40: 583-592.

16. Sangro B, Bilbao JI, Boan J, Martinez-Cuesta A, Benito A, et al. (2006) Radioembolization using 90Y-resin microspheres for patients with advanced hepatocellular carcinoma. Int J Radiat Oncol Biol Phys 66: 792-800.

17. Iñarrairaegui M, Thurston KG, Bilbao JI, D'Avola D, Rodriguez M, et al. (2010) Radioembolization with use of yttrium-90 resin microspheres in patients with hepatocellular carcinoma and portal vein thrombosis. J Vasc Interv Radiol 21 1205-1212.

18. Kennedy AS, McNeillie P, Dezarn WA, Nutting C, Sangro B, et al. (2009) Treatment parameters and outcome in 680 treatments of internal radiation with resin 90Y-microspheres for unresectable hepatic tumors. Int J Radiat Onco Biol Phys 74: 1494-1500.

19. Kulik LM, Carr BI, Mulcahy MF, Lewandowski RJ, Atassi B, et al. (2008) Safety and Efficacy of $90 \mathrm{Y}$ Radiotherapy for Hepatocellular Carcinoma With and Without Portal Vein Thrombosis. Hepatology 47: 71-81.

20. Raoul JL, Boucher E, Rolland Y, Garin E (2010) Treatment of hepatocellula carcinoma with intra-arterial injection of radionuclides. Nat Rev Gastroentero Hepatol 7: 41-49.

21. Salem R, Lewandowski RJ, Kulik L, Wang E, Riaz A, et al. (2009) Radioembolization results in longer time-to-progression and reduced toxicity compared with chemoembolization in patients with hepatocellular carcinoma. Gastroenterology 140: 497-507.

22. Hilgard P, Hamami M, Fouly AE, Scherag A, Müller S, et al. (2010) Radioembolization with yttrium-90 glass microspheres in hepatocellular carcinoma: European experience on safety and long-term survival. Hepatology 52: $1741-1749$.

23. Lewandowski RJ, Riaz A, Ryu RK, Mulcahy MF, Sato KT, et al. (2008) Optimization of Radioembolic Effect with Extended-shelf-life Yttrium-90 Microspheres : Results from a Pilot Study. J Vasc Interv Radiol 20: 1557-1563.

24. Becker S, Laffont S, Vitry F, Rolland Y, Lecloirec J, et al. (2008) Dosimetric evaluation and therapeutic response to internal radiation therapy of hepatocarcinomas using iodine-131-labelled lipiodol. Nucl Med Commun 29: 815-825.

25. Bretagne JF, Raoul JL, Bourguet $P$, Duvauferrier R, Deugnier $Y$, et al. (1998) Hepatic artery injection of I-131-labelled lipiodol - Part 2. Radiology 168: 547 550.

26. Raoul JL, Bourguet $P$, Bretagne JF, Duvauferrier R, Coornaert S, et al. Hepatic Artery Injection of I-131-labeled lipiodol - Part 1. Radiology 168: 541-545.

27. Raoul JL, Guyader D, Bretagne JF, Duvauferrier R, Bourguet P, et al. (1994) Randomized controlled trial for hepatocellular carcinoma with portal vein thrombosis: intra-arterial iodine-131-iodized oil versus medical support. J Nucl Med 35: 1782-1787.

28. Raoul JL, Guyader D, Bretagne JF, Heautot JF, Duvauferrier R, et al (1997) Prospective randomized trial of chemoembolization versus intraarterial injection of 131 I-labeled-iodized oil in the treatment of hepatocellular carcinoma. Hepatology 26: 1156-1161.

29. Lau WY, Leung TW, Ho SK, Chan M, Machin D, (1999) Adjuvant intra-arteria lipiodol-iodine-131 for resectable hepatocellular carcinoma: a prospective randomised trial. Lancet 353: 797-801.

30. Häfeli UO, Casillas S, Dietz DW, Pauer GJ, Rybicki LA, et al. (1999) Hepatic tumor radioembolization in a rat model using radioactive rhenium (186Re/188Re) glass microspheres. Int J Radiat Oncol Biol Phys 44: 189-199.

31. Bernal P, Raoul JL, Vidmar G, Sereegotov E, Sundram FX, et al. (2007) Intra- 
Citation: Burrill J, Hafeli U, Liu DM (2011) Advances in Radioembolization - Embolics and Isotopes. J Nucl Med Radiat Ther 2:107. doi:10.4172/21559619.1000107

Page 6 of 6

arterial rhenium-188 lipiodol in the treatment of inoperable hepatocellular carcinoma: results of an iaea-sponsored multination study. Int J Radiat Onco Biol Phys 69: 1448-1455.

32. Lambert B, Bacher K, Defreyne L, Gemmel F, Van Vlierberghe H, et al. (2005) 188Re-HDD/lipiodol therapy for hepatocellular carcinoma: a phase I clinical trial. Quality. 46: 60-66

33. Wunderlich G, Drews A, Kotzerke J (2005) A kit for labeling of [188 Re] human serum albumin microspheres for therapeutic use in nuclear medicine. App Radiat Isot 62: 915-918.

34. Liepe K, Brogsitter C, Leonhard J, Wunderlich G, Hliscs R, et al. (2007) Feasibility of high activity rhenium-188-microsphere in hepatic radioembolization. Jpn J Clin Oncol 37: 942-950.

35. J. Cwikla Initial study of radiological and clinical efficacy of i.a. radioembolisation (SIRT) using [188Re] HSA-spheres, in patients with advance primary or metastatic liver cancers. Department of Radiology, Hospital Ministry of Internal Affairs \& Administration, Warsaw. Unpublished data.

36. Häfeli UO, Saatchi K, Elischer P, Misri R, Bokharaei M, et al. (2010) Lung Perfusion Imaging with Monosized Biodegradable Microspheres. Biomacromolecules 11: 561-567.

37. Vente MA, de Wit TC, van den Bosch MA, Bult W, Seevinck PR, (2009) Holmium-166 poly(L: -lactic acid) microsphere radioembolisation of the liver: technical aspects studied in a large animal model. Eur radiol 20: 862-869.

38. Smits ML, Nijsen JF, van den Bosch MA, Lam MG, Vente MA, et al. (2010) Holmium-166 radioembolization for the treatment of patients with liver metastases: design of the phase I HEPAR trial. J Exp Clin Cancer Res 29: 70.

39. Sohn JH, Choi HJ, Lee JT, Lee JD, Kim JH, et al. (2009) Phase II study of transarterial holmium-166-chitosan complex treatment in patients with a single, large hepatocellular carcinoma. Oncology 76: 1-9. 